\title{
Metástase gigante de carcinoma papilífero
}

\author{
Giant metastasis of thyroid papillar carcinoma
}

Marcelo Benedito Menezes', Antonio Augusto Tupinambá Bertelli', Mauro Ajaj Saieg ${ }^{2}$, Tales Maciel de Camargo ${ }^{3}$, Antonio José Gonçalves'

\section{SUMÁRIO}

O carcinoma papilífero da tireoide, o mais comum deste órgão, geralmente se apresenta como lesões parenquimatosas pequenas e, eventualmente, com metástases cervicais numerosas, raramente volumosas. É descrito um caso raro de uma paciente do gênero feminino, 44 anos, com um tumor cervical anterior, nodular e volumoso há nove anos. Após o tratamento cirúrgico, o anatomopatológico mostrou tratar-se de metástases linfonodais de carcinoma papilífero. O objetivo deste estudo é relatar um caso clínico de apresentação incomum de carcinoma papilífero da tireoide, de diagnóstico inicial difícil e apresentando-se com metástases linfonodais volumosas. Arq Bras Endocrinol Metab. 2014;58(9):967-9

1 Departamento de Cirurgia de Cabeça e Pescoço, Irmandade da Santa Casa de Misericórdia de São Paulo (ISCMSP) São Paulo, SP, Brasil ${ }^{2}$ Departamento de Patologia, ISCMSP, São Paulo, SP, Brasil

${ }^{3}$ Faculdade de Ciências Médicas da Santa Casa de São Paulo (FCMSCSP), São Paulo, SP, Brasil

\section{SUMMARY}

Papillary thyroid carcinoma, the most common type of thyroid cancer is usually presented as small parenchymatous lesions and, eventually, with cervical lymph node metastasis, rarely voluminous. Here we describe a rare case of a 44-year-old woman presenting a visible anterior cervical tumor, nodullary and voluminous, for nine years. After surgical treatment, the anatomical pathology sample revealed that the mass was composed of several cervical lymph node metastatic lesions of a papillary thyroid carcinoma. We report the discovery of an uncommon papillary thyroid carcinoma manifestation, with a difficult initial diagnosis and presenting volu-

Correspondência para: Marcelo Benedito Menezes Rua Dr. Cesário Mota Jr., 112 01221-020 - São Paulo, SP, Brasil dr.mbmenezes@uol.com.br

Recebido em 30/Mar/2014 Aceito em 4/Maio/2014

DOI: 10.1590/0004-2730000003387 minous lymph node metastases. Arq Bras Endocrinol Metab. 2014;58(9):967-9

\section{INTRODUÇÃO}

$\mathrm{O}$ carcinoma papilífero da tireoide, a neoplasia mais comum deste órgão, abrange $75 \%$ (1) a $85 \%$ (2) dos 60.220 novos casos estimados de câncer de tireoide nos Estados Unidos em 2013. Desses tumores da tireoide, $75,24 \%$ ocorrem em mulheres (3), normalmente em jovens adultos. A idade média de diagnóstico do câncer de tireoide é de $54 \mathrm{em}$ homens e $48 \mathrm{em}$ mulheres (4). Em pacientes com câncer papilífero da tireoide, há alta incidência de metástases cervicais ao primeiro diagnóstico, dependendo não só do estadiamento como também de qual método é utilizado na pesquisa por potenciais metástases (5). Metástases para linfonodos cervicais ocorrem em 20\% a $50 \%$ dos carcinomas papilíferos de tireoide no exame anatomopatológico de peças cirúrgicas (6) e raramente são volumosas. O objetivo do presente trabalho é relatar um caso de apresentação incomum de carcinoma papilífero da tireoide, de diagnóstico inicial difícil e apresentando-se com metástases linfonodais volumosas, atendido no Serviço de Cirurgia de Cabeça e Pescoço da Santa Casa de São Paulo.

\section{RELATO DE CASO}

Mulher, 44 anos, branca, solteira, natural e procedente de Santo André/SP, foi admitida no Pronto-Socorro municipal de Santo André devido à presença de tumor cervical que apresentou ulceração, sangramento e extravasamento de conteúdo amarelo (Figuras l e 2). Após cuidados iniciais, foi encaminhada ao Serviço de Cirurgia de Cabeça e Pescoço da Santa Casa de São Paulo. Queixava-se de nódulo cervical anterior há nove anos, inicialmente de crescimento rápido, permanecendo com volume estável nos últimos dois anos. Referia 
ser o nódulo doloroso e incômodo no que diz respeito à mobilidade e estética. Há cinco anos procurou serviço médico, sendo sugerida a não intervenção cirúrgica. Paciente negava sintomas compressivos e apresentava hirsutismo desde os 20 anos de idade e sinais de virilizacão, comprovados ao exame clínico, frequência e volume menstrual irregulares e hipertensão arterial sistêmica controlada com uso de captopril $25 \mathrm{mg} /$ dia.

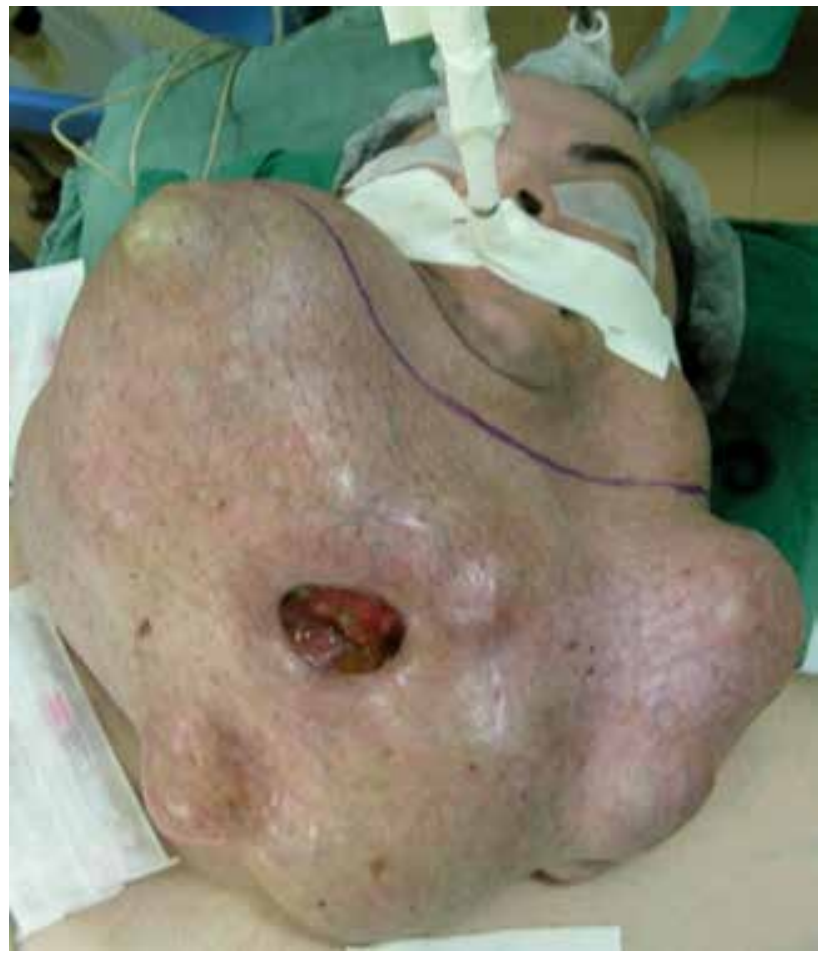

Figura 1. Apresentação do tumor ulcerado em visão frontal.

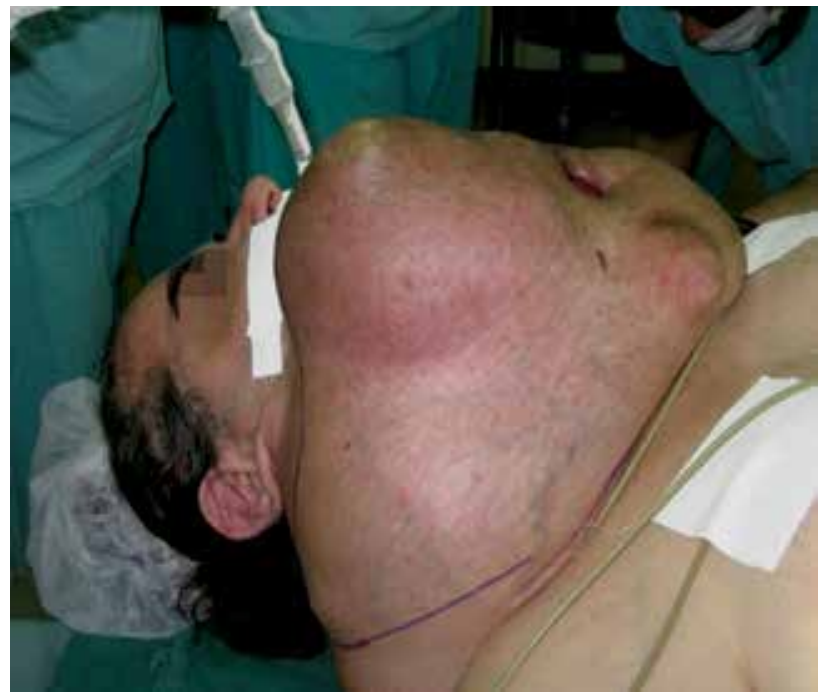

Figura 2. Apresentação do tumor em visão de perfil.
A orofaringolaringoscopia e a rinoscopia foram normais. No exame físico, a paciente era brevilínea, obesa, normotensa, eufônica, apresentava exame neurológico normal e não possuía alterações respiratórias. A dosagem de hormônios tireoidianos foi normal e a radiografia de tórax não apresentava alargamento mediastinal.

O diagnóstico inicial foi de bócio gigante, sendo indicado tratamento cirúrgico. A paciente foi submetida à cervicotomia exploradora com ressecção de grande área de pele que recobria a lesão. No intraoperatório, notou-se que a totalidade da lesão encontrava-se fora da loja tireoidiana, anteriormente aos músculos pré-tireoidianos, sendo ressecada sem intercorrências, exceto pela secção do músculo esternocleidomastóideo, com posterior ressutura, e ligadura das veias jugulares anteriores e externas. A peça cirúrgica pesou $7,2 \mathrm{~kg}$ e mediu $37 \mathrm{~cm} \times 30 \mathrm{~cm}$ (Figura 3). Durante a cirurgia não foi localizado nenhum nódulo tireoidiano palpável, sendo encerrada sem a ressecção da glândula. O exame anatomopatológico de congelação não foi conclusivo, apesar de sugerir presença de tecido tireoidiano.

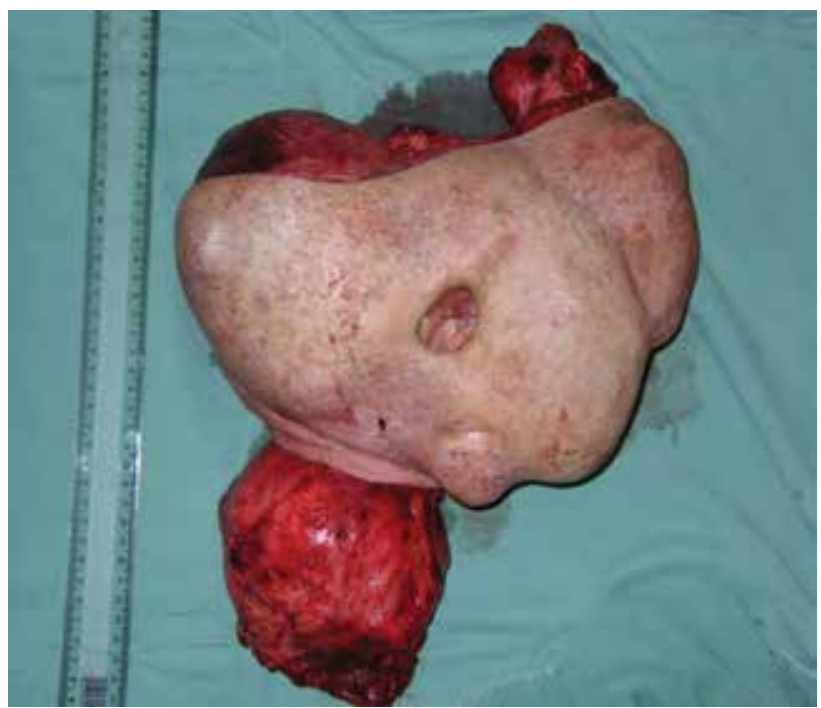

Figura 3. Peça cirúrgica de $7,2 \mathrm{~kg}$, medindo $37 \mathrm{~cm}$ x $30 \mathrm{~cm}$.

Paciente evoluiu sem complicações. O anatomopatológico mostrou tratar-se de metástases linfonodais de carcinoma papilífero. Indicada tireoidectomia total onde foram encontrados dois focos de carcinoma papilífero menores que $2 \mathrm{~cm}$. No pós-operatório de três meses, observou-se a presença de linfonodos palpáveis na região cervical, sendo realizado novo esvaziamento à esquerda. Paciente evoluiu com depressão, necessitando de medicação específica, mas com bom controle hormonal e programação de dose de iodo 131. Após 
sete meses da última cirurgia, paciente faleceu em casa, sem causas definidas.

\section{DISCUSSÃO}

O carcinoma papilífero costuma evoluir de forma benigna, apresentando bom prognóstico quando submetido a tratamento adequado e intervenção precoce. Mostramos um caso de apresentação atípica, primeiro pelo volume descomunal das metástases cervicais desproporcional à pequena agressividade local e a distância. Apesar de volumosos, os linfonodos metastáticos não invadiam as estruturas adjacentes e também não causavam sintomas compressivos esperados, exceto incômodo relativo pelo próprio volume e peso. Sem dúvida houve falha na interpretação diagnóstica inicial, quando acreditamos tratar-se de uma lesão própria da glândula. Ao rever os exames tomográficos (Figura 4), notamos a musculatura tão delgada que não podíamos individualizá-la, dificultando a percepção de uma glân-

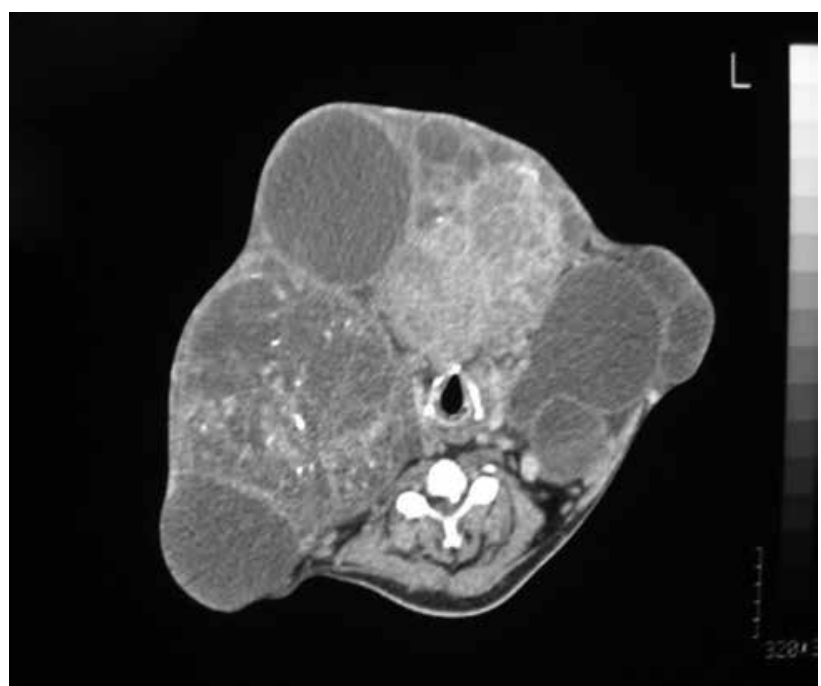

Figura 4. Imagem de tomografia computadorizada evidenciando nódulos fora da loja tireoidiana. dula tireoide pouco alterada e a presença dos nódulos fora da loja tireoidiana. A necessidade de três cirurgias subsequentes para obter o controle macroscópico da neoplasia não parece ter trazido maiores prejuízos à paciente, assim como não estabelecemos uma relação direta do óbito da paciente, seja com a neoplasia ou com o processo terapêutico.

\section{CONCLUSÃO}

O caso apresentado representa uma evolução atípica do carcinoma papilífero da tireoide, tanto no que diz respeito à sua apresentação clínica quanto ao seu desfecho.

Declaração: os autores declaram não haver conflitos de interesse científico neste estudo.

\section{REFERÊNCIAS}

1. Sakorafas GH, Sampanis D, Safioleas M. Cervical lymph node dissection in papillary thyroid cancer: current trends, persisting controversies, and unclarified uncertainties. Surg Oncol. 2010;19(2):e57-70.

2. Hundahl SA, Fleming ID, Fremgen AM, Menck HR. A National Cancer Data Base report on 53,856 cases of thyroid carcinoma treated in the U.S., 1985-1995. Cancer. 1998;15;83(12):2638-48.

3. Jemal A, Siegel R, Xu J, Ward E. Cancer statistics, 2010. CA Cancer J Clin. 2010;60(5):277-300.

4. Howlader N, Noone AM, Krapcho M, Garshell J, Miller D, Altekruse SF, et al. SEER Cancer Statistics Review, 1975-2011, National Cancer Institute. Bethesda, MD. Disponível em: http://seer.cancer. gov/csr/1975_2011/, based on November 2013 SEER data submission, posted to the SEER web site, April 2014. Acesso em: $15 \mathrm{dez}$, 2014.

5. Sivanandan R, Soo KC. Pattern of cervical lymph node metastases from papillary carcinoma of the thyroid. $\mathrm{Br} \mathrm{J}$ Surg. 2001;88(9):1241-4.

6. American Thyroid Association (ATA) Guidelines Taskforce on Thyroid Nodules and Differentiated Thyroid Cancer, Cooper DS, Doherty GM, Haugen BR, Kloos RT, Lee SL, Mandel SJ, et al. Revised American Thyroid Association management guidelines for patients with thyroid nodules and differentiated thyroid cancer. Thyroid. 2009;19(11):1167-214. 